\title{
Pulse Acoustic Technology lor Measurement of Charge Distroibution in Dielectric Materials for Spacecraft
}

\author{
Tatsuo Takada and Yasuhiro Tanaka \\ Musashi Institute of Technology \\ Electronic Measurement Laboratory, Musashi Institute of Technology, \\ 1-28-1, Tamazutsumi, Setagaya-ku, Tokyo, 158-8557, Japan \\ Phone \& Fax : $+81-3-5707-2160$ \\ E-mail : takada@yc.musashi-tech.ac.jp
}

\begin{abstract}
Recently, some accidents in spacecraft due to the charging up by irradiation of charged particle have been reported. Some of them are caused by surface discharge normally happens in plasma environment of space. Some others seem to be caused by discharge due to an accumulation of charge in bulk of materials in relatively higher altitude environment. To investigate the charge accumulation in bulk of materials, we have been developing the internal charge measurement system. The internal charge accumulation in dielectric materials under irradiation of artificial radioactive ray is observed using the developed systems, which are so called PEA and PWP methods based on acoustic measurement techniques. The developed system is applicable to measure the charge distribution in dielectrics in air atmosphere or vacuum environment under electron beam, proton beam and gamma ray irradiations. Using the systems, we carried out the measurements of bulk charge distributions in polymeric films and glass materials. In this report, summary of some typical measurement results are introduced following the brief explanation of measurement principles.
\end{abstract}

\section{Introduction}

In space environment, especially at higher altitude such as GEO, spacecraft is exposed under high-energy radioactive rays such as electrons, protons and/or gamma ray. When the radioactive rays from solar flares are irradiated to the insulating materials of spacecraft such as heat control polymer sheets or cover glasses of solar battery, it is said that the charges accumulate on the surface and/or in the bulk of them and sometimes they cause to the electrical discharge with serious damage in the electric devices of spacecraft [1,2].

The accidents in such environment are though that they must be deeply related with an interaction between dielectric materials and high-energy charged particles composed of electrons and protons, which are suddenly scattered accompanied with solar flares. However, the mechanism how such high energy particles give serious damages to the spacecrafts has not been clear yet. Therefore, it is necessary to investigate the relationship between the accidents and the accumulation of charge by irradiation of radioactive rays. However, it had been difficult to measure the charge distribution in bulk of insulating materials directly.

While numerical simulations based on data of surface potential measurements for charged dielectrics by irradiation of high energy particles have been attempted to understand the phenomenon [3], it seems to be difficult to study the phenomenon quantitatively. Therefore, we have been tried to develop some kinds of measurement systems to observe the bulk charge distribution in dielectrics under irradiation of high energy electron beam in quasi-space environment $[4,5]$.

Advanced measurement techniques for the electric charge accumulation in dielectrics, so called PEA and PWP methods, have been mainly developed for measurement of high voltage equipments [6]. In the beginning stage of developing the PEA method in 1980's, we tried to observe the electric charge accumulation in dielectrics irradiated by high energy electron beam $[4,5]$, since it was easy to get a relatively large acoustic signal from accumulated charge in dielectrics in which a lot of electric charge was stored. Advanced measurement techniques have been applied for evaluating the various dielectrics such as polymer, glass and photo-conducting materials with various shapes such as sheet, plate and co-axial cable geometries.

As shown in this report, these advanced measurement techniques could be applied to the basic investigation on space charge accumulation exposed by high-energy radioactive rays, especially electron beam, gamma ray and proton irradiation, for various environmental conditions in air or vacuum atmospheres, at room temperature or elevated temperature. Many results shown in this report suggest that the techniques are effective to investigate the internal charge accumulation in dielectric materials at space environmental conditions.

\section{Measurement of Internal Nlectric Charge Distribution}

We have developed two methods, which is so called PEA (pulsed electro-acoustic) and PWP (pressure wave propagation) methods to measure the internal electric charge distribution in insulating materials. Here, the principles of those methods are described briefly. 
Primeiple of PRE method

The PEA method is one of widely used techniques to measure the charge distribution in dielectrics. A schematic diagram of principle for the PEA method is shown in Figure 1. Consider a sheet sample with thickness of $d$ and charge distribution $\rho(z)$. A pulsive electric field $E_{p}(t)$ is applied extemally to the sample and it induces a perturbation force on each charge. This force causes the charge to move slightly. This movement generates an acoustic wave that is proportional to the charge distribution in the sample. A piezoelectric transducer is used to detect the acoustic wave by transforming the acoustic wave into an electric signal. The detail of the measurement is described elsewhere [7]. In the PEA measurement system, since the detector of the piezo-electric transducer is completely shielded and it separated from the sample, we can measure the charge distribution with low electric noise. Because it is necessary to apply the high voltage pulse to the sample to obtain a sufficient level of electrical signal, however, it is difficult to apply the method in vacuum environment. If the high voltage pulse is applied to the sample in vacuum environment, it is expected that an electrical discharge based on Paschen's law must happen. Therefore, it is necessary to improvement the system to use it as the monitoring system in space environment. At present time, we have mainly used the system for the fundamental research works to investigate the charge accumulation after irradiation at laboratory.

\section{Principle of PW method}

The PWP method is another major technique to measure the charge distribution in dielectrics. A schematic diagram of principle for PIPWP (Piezo-Induced PWP), which is one of PWP method, is shown in Figure 3. In this technique, the acoustic wave acts as a "probe" for charge detection. Since the charge accumulated in bulk moves when the acoustic wave propagates through it, the movement causes a change of induced surface charge on the electrodes. Therefore, the time dependent signal of displacement current of extemal circuit indicates the charge distribution in the sample. By measuring the displacement current, we can obtain the charge distribution in bulk of sample. The details of the measurement are described elsewhere [7]. Since the electric pulse voltage, which is applied to a piezo-electric transducer to generate the pulse acoustic wave, is not so high in the PWP system, this method is applicable to the measurement under vacuum condition. Therefore, this method may be used as a monitor in spacecraft. In this measurement system, since the sensitive amplifier is connected to the sample irradiated by the radioactive rays, it is easily to be affected damage by the electric noise. Therefore, it is necessary to make some improvement to use it as the monitoring system in space environment.

\section{Comparison of both measurement results}

A PMMA (poly-methyl-methacrylate) sample with thickness of 500 um is measured using both PEA and PWP methods for comparison. The sample is irradiated by electron beam with energy of $200 \mathrm{keV}$ and current density of $1.0 \mathrm{nA} / \mathrm{cm}^{2}$ for 3 hours. The space charge distributions in the short circuited sample are shown in Figure 2 (a) and 4 (a) observed using PEA and PWP methods, respectively. The results obtained using both methods are almost the same each other. Judging from a valley is found at $z=160 \mu \mathrm{m}$, the irradiated electrons must be concentrating there. The maximum penetrate depth of irradiated electrons is about $400 \mu \mathrm{m}$. Surface charges induced by bulk charge show two peaks at $z=$ 0 and $500 \mu \mathrm{m}$ in the figure.

As described in explanation of principle, surface charge density $\sigma$ should be a $\delta$ function if one expresses it in the coordinator of volume space charge density. However, due to the finite resolution of detecting device and the finite width of the generated pulse, it is impossible to obtain this ideal result. Consequently, the induced surface charges are expressed as a peak and a valley in the coordinate axis of space charge density as shown in Figures 2 (a) and 4 (a). In other words, the widths of the peak and the valley reflect the resolution of the measurement system. The narrower width of it means the higher resolution. We define the width at half height of the peak as the resolution. Since the half height widths observed using both systems are about $16 \mu \mathrm{m}$, the resolutions of them are almost the same each other.

The distributions of electric field $E(z)$ and potential $V(z)$ are demonstrated in Figure $2(b), 2(c)$ and Figure $4(b), 4(c)$ observed using the PEA and the PWP methods, respectively. The distributions of electric field $E(z)$ along $z$ direction are calculated by integration of $\rho(z) / \varepsilon_{\mathrm{r}} \varepsilon_{0}$. The distributions of electric potential $V(z)$ are obtained by integration of $E(z)$. As shown in the figures, electric field vanishes at $z=190 \mu \mathrm{m}$ in the sample. The distributions of electric potential in the sample look like triangle shapes as illustrated in Figure $2(\mathrm{c})$ and $4(\mathrm{c})$. Since the both surfaces of the sample are connected to the ground level, the electric potential should be zero at each electrode. Therefore, the peak of the triangle located at $z=190 \mu \mathrm{m}$ is corresponding to the position where $E(z)$ is zero.

The results of the distributions of electric charge density $\rho(z)$, electric field $E(z)$ and potential $V(z)$ measured using both methods seems to be almost the same. It means that both of the measurement methods can be applicable to measure the charge distribution in bulk of insulating materials.

\section{Observation of Dynamic Charge Behavior}

It has been known that the electrical breakdown of insulating materials is strongly affected by both of the formation of the space charge and the local electric field intensity in bulk of materials. When a high voltage is applied to an insulating material, the space charge gradually formed in the bulk of it. To observe the formation of the space charge, we measured the space charge distribution as a function of position $z$ and voltage application time $t$. The advanced PEA system is developed to measure the space charge distribution in thin polymeric film with high spatial resolution (about $10 \mu \mathrm{m}$ ) under the high electric field (about $5 \mathrm{MV} / \mathrm{cm}$ ) with short intervals $(0.5 \mathrm{~ms}$ ) for long time (over $10 \mathrm{~min}$.). Using 


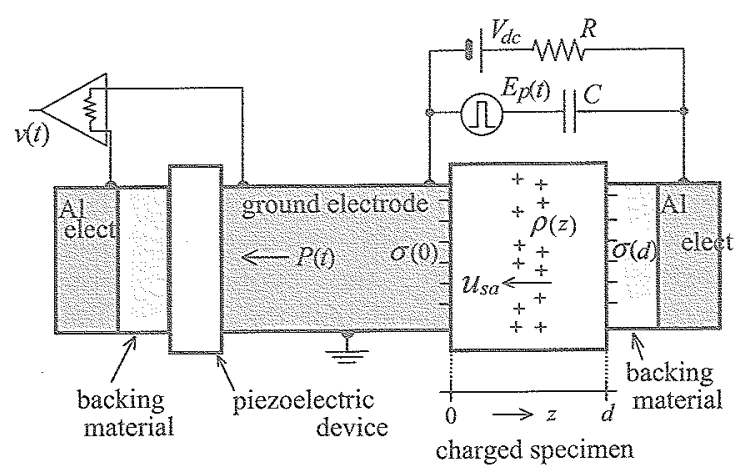

Figure 1. Block diagram of the PEA method for measuring the space charge distribution in solid dielectrics

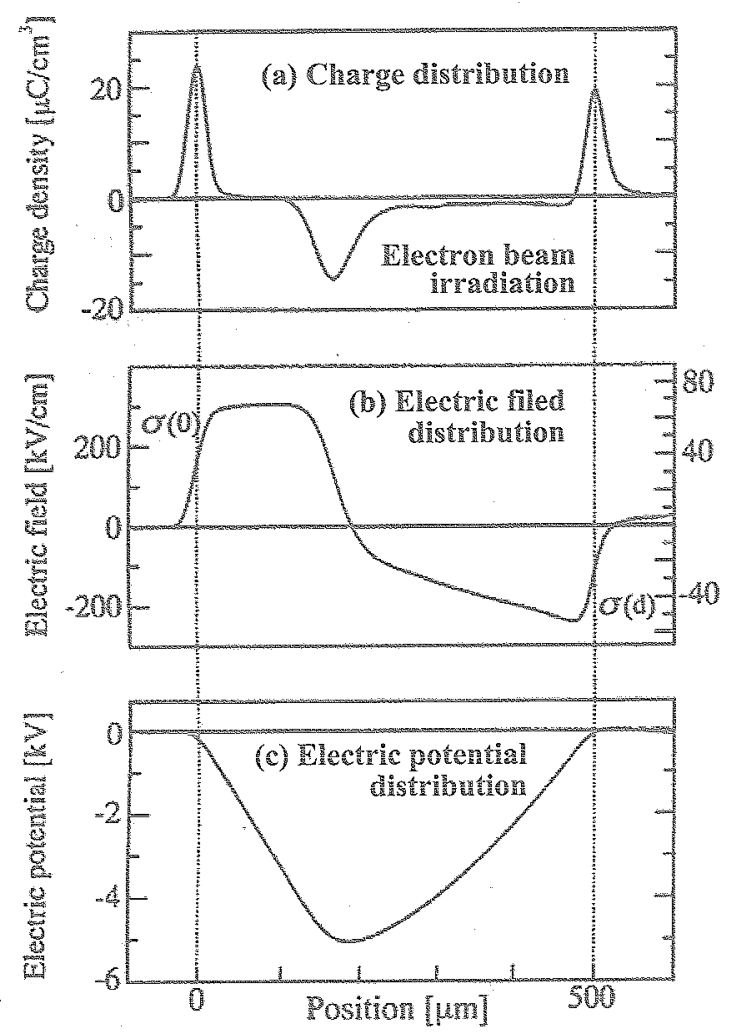

Figure 2. Space charge measurement results in e-beam irradiation PMMA obtained by using the PEA method. (a) space charge distribution, (b) electric field distribution and (c) electric potential distribution.

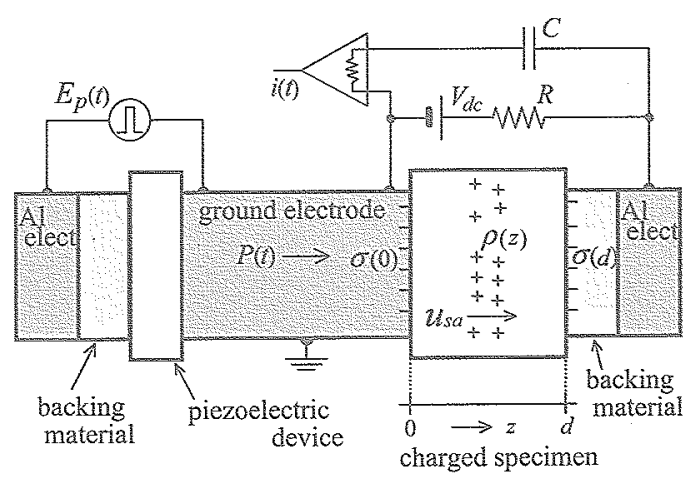

Figure 3. Block diagram of the PWP method for measuring the space charge distribution in solid dielectrics

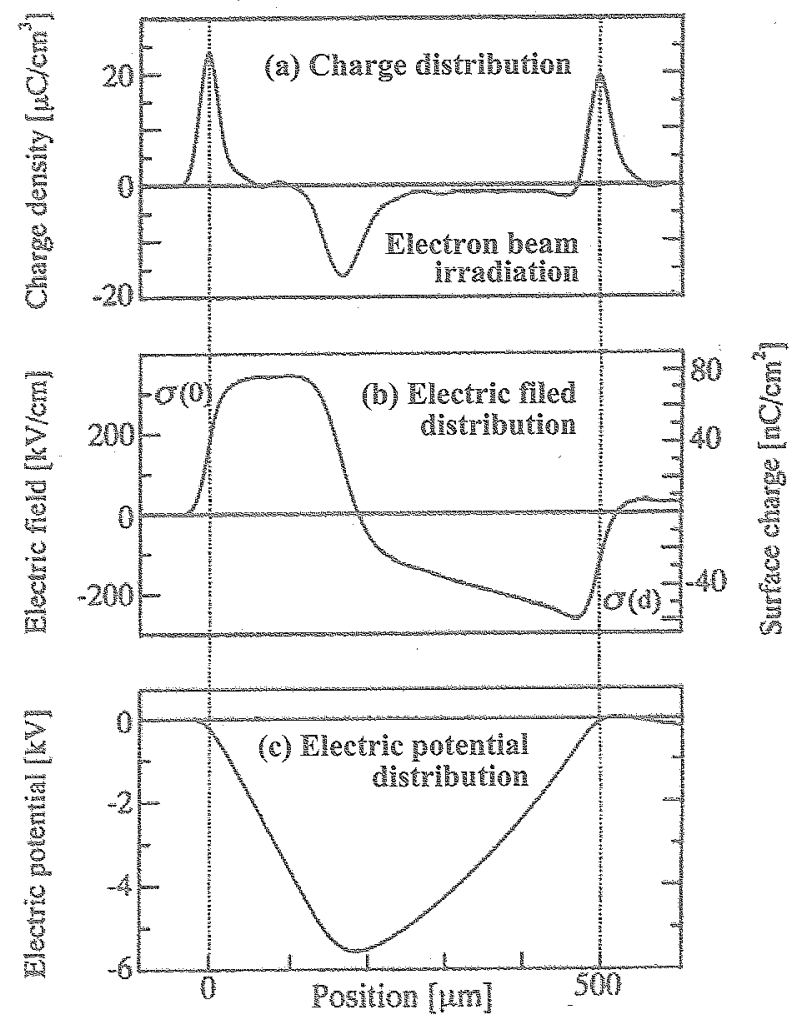

Figure 4. Space charge measurement results in e-beam irradiation PMMA obtained by using the PWP method. (a) space charge distribution, (b) electric field distribution and (c) electric potential distribution.

this system, we observed the space charge dynamics in low-density polyethylene (LDPE) at and around the breakdown field [8].

\section{Results and discussion}

Figure 5 shows a typical measurement result observed using the advanced PEA system by applying $50 \mathrm{kV}$ to the LDPE sample. The time dependent space charge profiles are described using color charts as shown in Figure 5 . In the color chart, the red and the blue colors stand for the positive and the negative charge densities, respectively. The color bar described beside the data shows the scale of the charge density for the color chart. In this figure, the vertical axes for the time before and after breakdown are described with unit of minute and second, respectively. The horizontal axis stands for the position in the bulk of the sample. Left and right vertical lines show the position of the cathode and the anode electrodes, respectively. 
As shown in Figure 5, the large amount of positive packet charge was injected from the anode after voltage application. The injected positive packet moved towards the cathode side with increase of voltage application time. The movement, however, gradually became to be slow and finally it stopped at the middle of the bulk. At about 20 minutes after voltage on, the breakdown happened in the sample. After the breakdown, the positive charge split into two parts and they moved towards each counter electrode. About 40 seconds after the breakdown, the positive charge packets disappeared.

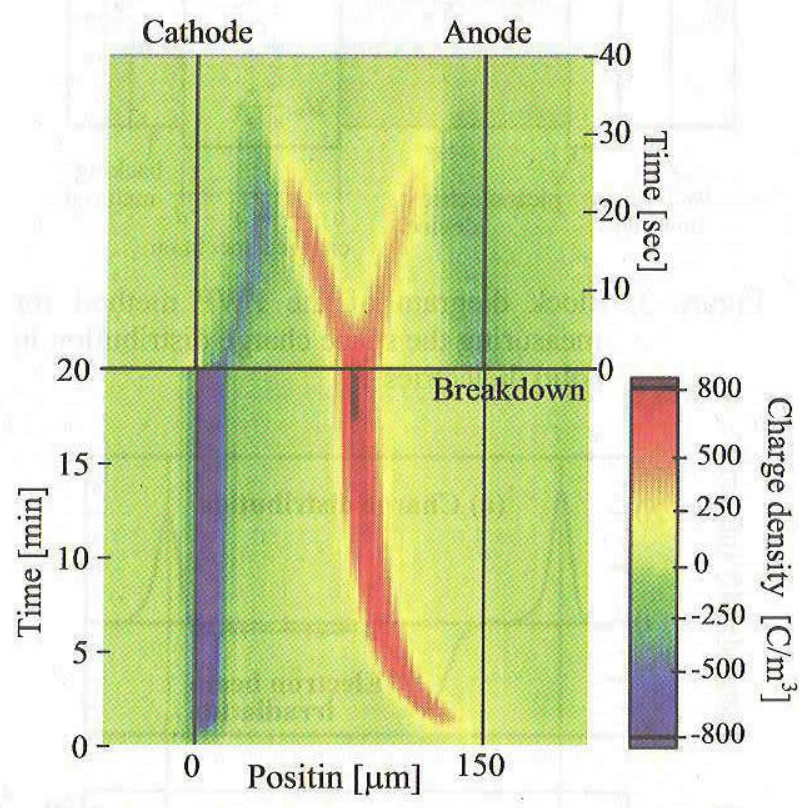

Figure 5. Time dependent space charge profiles. The red and the blue colors for the positive and the negative charge densities, respectively. The horizontal axis stands for the position in the bulk of the sample. The vertical axes stand for the time before and after breakdown. Left and right vertical lines show the position of the cathode and the anode electrodes, respectively.
Figures 6 (a) and (b) show the time dependent space charge distribution and calculated electric field distribution during the voltage application, respectively. It is found that the electric fields are increasing with the movement of the positive packet charges in all results. When the positive packets reach the stoppage position, the electric fields at the positions become maxima. The maximum electric fields are about $5.5 \mathrm{MV} / \mathrm{cm}$. Figures 6 (c) and (d) show the time dependent space charge and calculated electric field distributions after the breakdown. Immediately after the breakdown, the electric field in the bulk generated by the existence of the injected positive charge is about $3 \mathrm{MV} / \mathrm{cm}$.

We observed space charge profiles in LDPE under various electric fields, 0.5 to $4.0 \mathrm{MV} / \mathrm{cm}$, before and after breakdowns. It is found that the maximum injection depth just before the breakdown is inversely proportional to the initially applied field. On the other hand, it is found no matter how much electric field is initially applied to the sample, the breakdown strength of local electric field just before the breakdown are almost the same value of about 5.2 $\mathrm{MV} / \mathrm{cm}$. Reasons for above mentioned curious phenomena have been still investigated. However, the results are significant data, which may be one of key to show the relationship between the breakdown and the space charge accumulation.
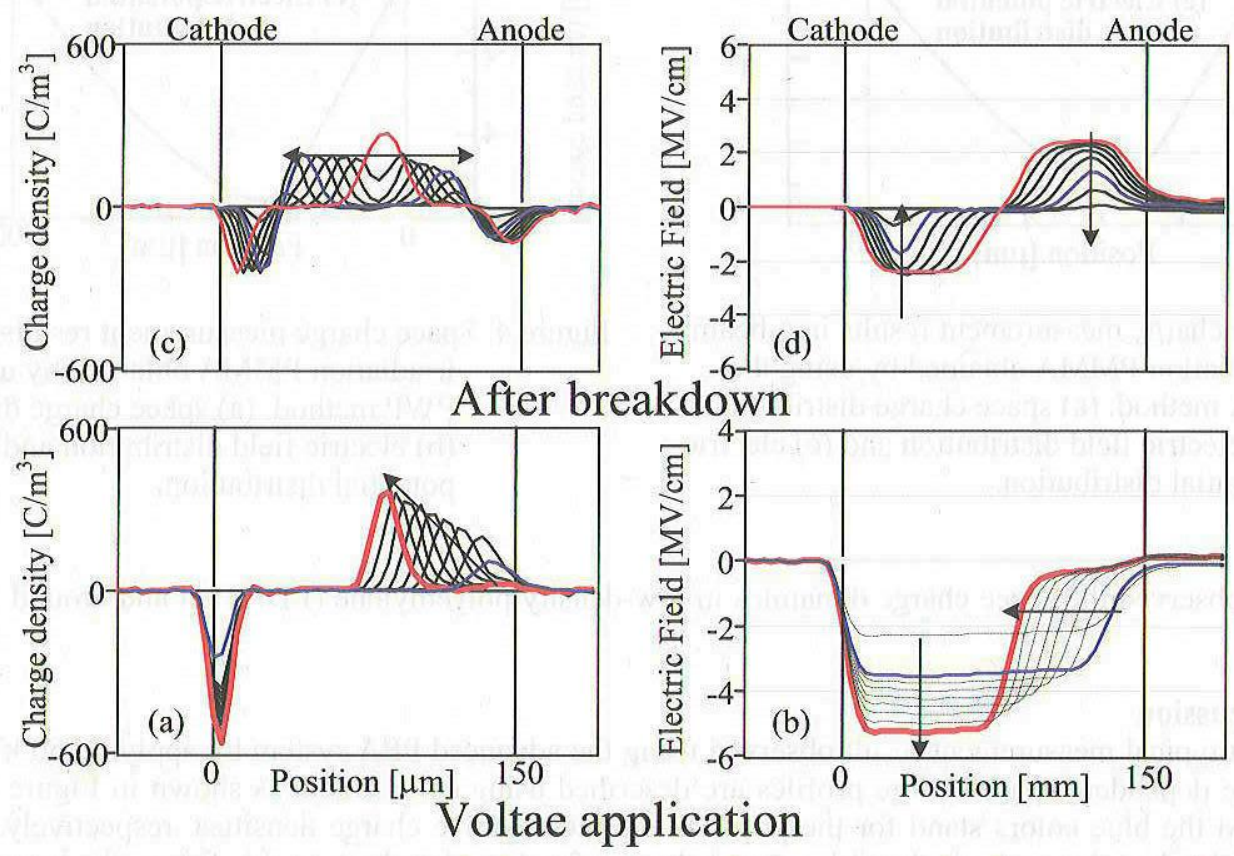

Figure 6. Typical space charge and electric field profiles in $150 \mu \mathrm{m}$-thick LDPE applied $50 \mathrm{kV}$.

(a) Time dependence of space charge profiles before breakdown.

(b) Time dependence of electric field distributions before breakdown.

(c) Time dependence of space charge profiles after breakdown.

(d) Time dependence of electric field distributions after breakdown. 
4. Internal Charge Accumulation by Electron Beam Irradiation

It is necessary to measure the charge distribution under electron beam irradiation when we would like to investigate on the spacecraft charging. Therefore, we attempted to develop a new designed PWP system to measure the charge distribution under electron beam irradiation [9].

\section{Experimental set up and electron beam} irradiation

Figure 7 shows a schematic diagram of the newly developed apparatus for a real-time measurement system under electron beam irradiation. New apparatus must have a window for the irradiation of electron beam to the sample. Therefore, a window is made on the topside electrode of the apparatus as shown in Figure 7. To obtain the electric signal from the bottom side of the sample, a glass plate is inserted between the sample and the piezo-electric device. This glass plate is used to isolate the bottom side of the sample from the grounded level. As the aluminum electrode is evaporated on the bottom side of the glass plate for the shielding, the sample is completely covered by the grounded shield. The glass plate also has an evaporated electrode on the topside surface and it is connected to the detecting amplifier.

The electron beam irradiation was carried out in air atmosphere using the EC300 (Iwasaki Electric Co. Ltd.,) with the energy of $230 \mathrm{keV}$ and the beam current of $10 \mathrm{~mA} / \mathrm{cm}^{2}$ for 5 minutes. The electrons are accelerated in the vacuum chamber and they come down through the titanium foil of $12.7 \mu \mathrm{m}$ thick. After passing through the foil, the electrons arrive at the surface of the sample through the air gap of about $5 \mathrm{~cm}$.

\section{Charging Process}

Figures 8 (a) shows the time dependence of the charge distributions in PMMA of $510 \mu \mathrm{m}$ thick during the electron beam irradiation. The results shown in these figures are obtained at 30 seconds before the irradiation and every 1-minute during the irradiation. Within 1 minute after start of the irradiation, no remarkable peak was observed in the charge distribution. After 1 minute from the start of the irradiation, a negative peak appeared at the depth of about $200 \mu \mathrm{m}$ from the irradiation surface. The peak height gradually increased during the irradiation and finally it reached the value of about $-33 \mu \mathrm{C} / \mathrm{cm}^{3}$. Judging from this result, the deepest position of the electron beam penetration seems to be at $315 \mu \mathrm{m}$ from the irradiation surface.

The electric field distributions shown in Figure 8 (b) were calculated using the charge distributions shown in Figure 8 (a). It is clearly shown that the direction of the electric field distribution is separated into two zones. The zones of the left and right hand sides are the positive and the negative electric fields, respectively. The negative electric field gives a force towards the right-hand side to the injected electrons. In other words, the negative electric field works as a

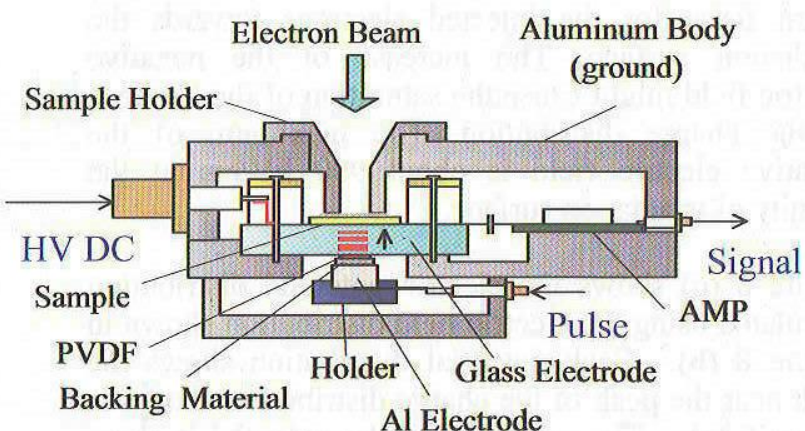

Figure 7. PWP measurement system for measuring the internal charge accumulation under electron beam irradiation at atmosphere

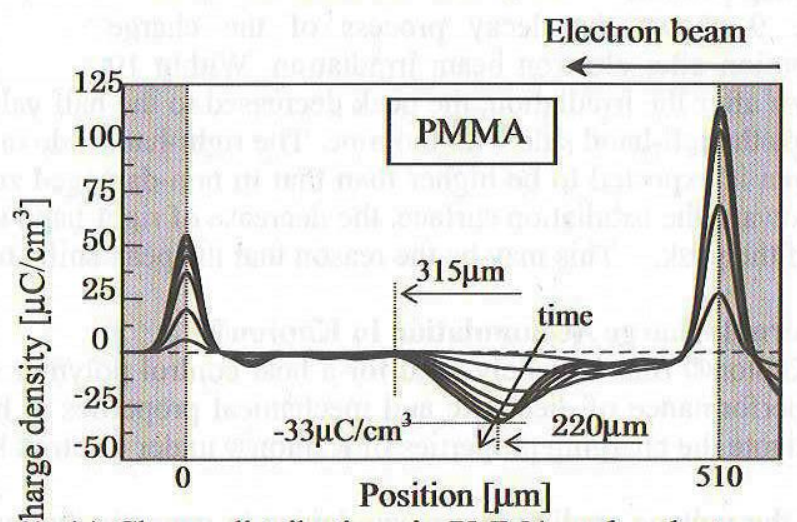

(a) Charge distributions in PMMA under electron beam irradiation

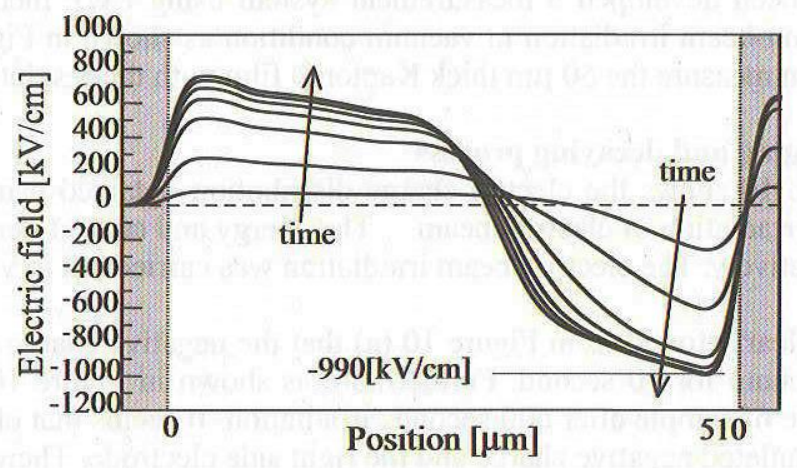

(b) Electric field distribution in PMMA under electron beam irradiation

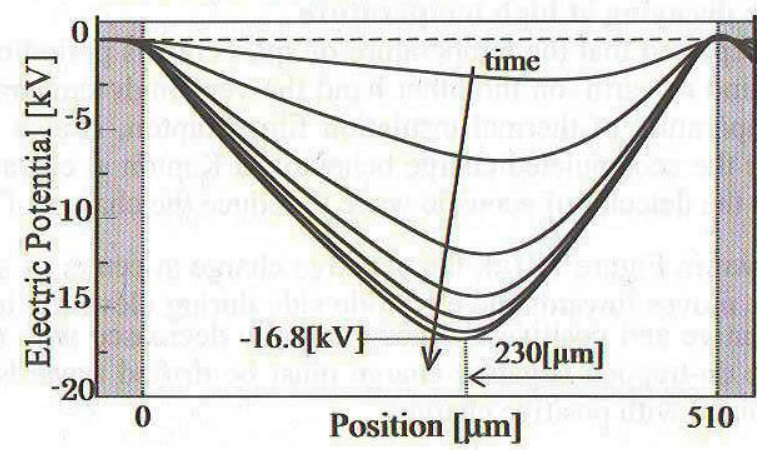

(c) Electric potential in PMMA under electron beam irradiation

Figure 8. Time dependence of

(a) space charge distribution,

(b) electric field distributions,

(c) electric potential distributions

in PMMA sample during an electron beam irradiation 
retum force for the injected electrons towards the iradiation surface. The increase of the negative electric field might cause the saturation of the increase of the charge distribution. The maximum of the negative electric field is about $990 \mathrm{kV} / \mathrm{cm}$ at the vicinity of iradiation surface.

Figure 8 (c) shows the electric potential distribution calculated using the electric field distribution shown in Figure 8 (b). Each potential distribution shows the peak near the peak of the charge distribution shown in Figure 8 (a). The maximum of the potential is about $16.8 \mathrm{kV}$.

\section{Desaying procas}

Figure 9 shows the decay process of the charge

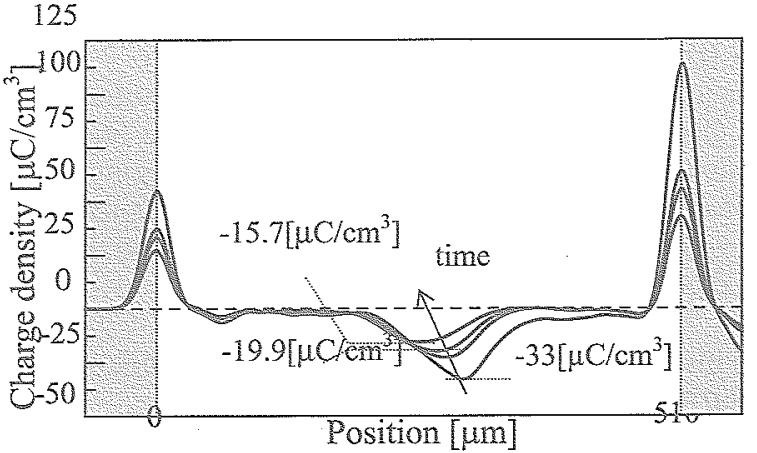

Figure 9. Time dependence of space charge distribution in PMMA sample after an electron beam irradiation distribution after electron beam iradiation. Within 10 minutes after the iradiation, the peak decreased to the half value of the initial. It is found that the peak gradually shifts towards the left-hand side with the time. The right-hand side in this figure may be the damaged zone and the mobility in this area is expected to be higher than that in non-damaged zone. Since the electrons are expected to escape from the bulk toward the irradiation surface, the decrease of righthand side of the peak should be faster than that of the left-hand side of the peak. This may be the reason that the peak shifts towards the left-hand side gradually.

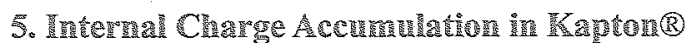

The Kaptoni fim is widely used for a heat control polymer sheets of spacecraft in space environment because of its high performance of dielectric and mechanical properties at high temperature. Therefore, it seems to be important to investigate the charging properties of Kapton(B) under electron beam irradiation in vacuum condition.

Since the voltage applied to a piezo-device to generate the pulse acoustic wave is lower than the minimum discharge voltage of Paschen's low, we don't need to wory about discharge in the system even in vacuum environment. We have been developed a measurement system using PWP method to measure the intemal charge in dielectrics under electron-beam irradiation in vacum condition as shown in Figure 7. The newest one has a resolution of 5 um. So, we can measure the 50 um thick Kaptone inm with the resolution of $10 \%$.

\section{Charging and decaying process}

Figure 10 shows the electic charge distribution obtained using PEA system in Kapton\& film (125 um in thickness) after irradiation of electron beam. The energy and current density of the electron beam were $70 \mathrm{keV}$ and $0.05 \mathrm{~mA} / \mathrm{cm}^{2}$, respectively. The electron beam inadiation was carried out in vacuum of $1.2 \times 10^{-4} \mathrm{~Pa}$.

It is clearly found from Figure 10 (a) that the negative charge is accumulated in Kapton $($ by electron beam irradiation in vacuum for 10 second. Furthemore as shown in Figure 10 (b), negative and positive charges are observed in the middle of sample after 600 seconds irradiation. It seems that electric field intensity is enhanced at the zone between the accumulated negative charge and the right side electrode. Therefore the trapped electron was drifted by the local electric field. Consequently the positive charge was appeared at the next to the negative charge.

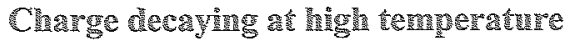

It was reported that the temperature of spacecrat is periodically changed from the very low temperature of $-180^{\circ} \mathrm{C}$ at the behind of earth, on the other hand the very high temperature of $+150^{\circ} \mathrm{C}$ at the face to solar side [10]. Therefore the temperature of themal insulation fim, Kapton, is also changed. Consequently the PEA system was improved to observe the accumulated charge behavior in Kapton at elevated temperature [11]. In this system, the LiNbO $_{3}$ crystal is used as the detector of acoustic wave to reduce the change of sensitivity by increase of temperature.

As shown in Figure 11 (a), the negative charge at center of sample decreased beween $30^{\circ} \mathrm{C}$ and $120^{\circ} \mathrm{C}$. The negative charges moves towards the electrode side during elevating temperature. On the other hand, as shown in Figure 11 (b), the negative and positive charges gradually decreased with elevated temperature between $30^{\circ} \mathrm{C}$ and $120^{\circ} \mathrm{C}$. It means that the de-trapped negative charge must be drifted towards the area of positive charge accumulation and it must be recombined wih positive charge. 

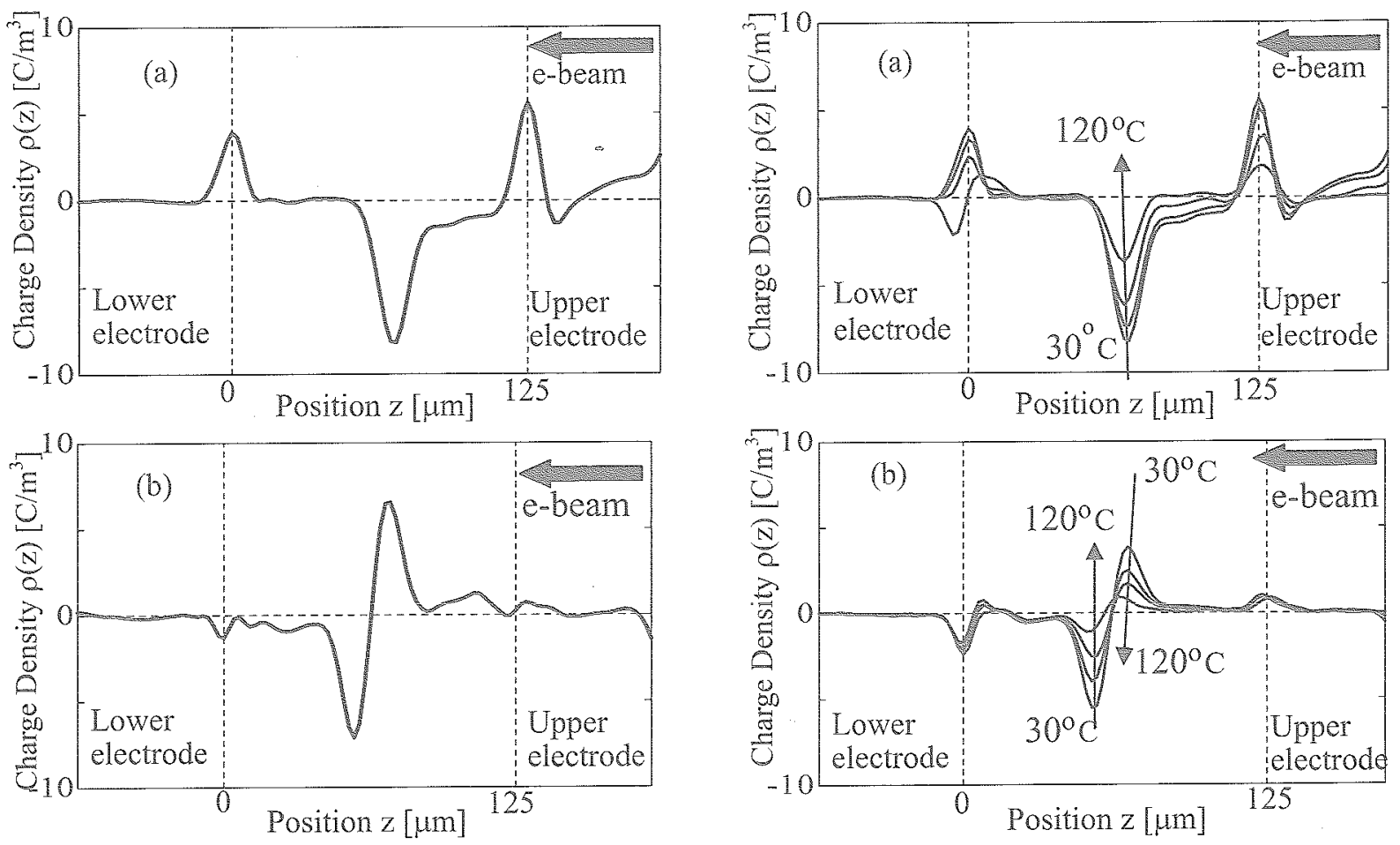

Figure 10. Electric charge distribution in Kapton $($ Q film after irradiation of electron beam obtained by using PEA system

(a) exposing time $10 \mathrm{sec}$.

Figure 11. Electric charge distribution in Kapton( $\mathrm{B}$ film after irradiation of electron beam obtained by using PEA system under elevating temperature

(a) exposing time $10 \mathrm{sec}$.

(b) exposing time $600 \mathrm{sec}$

\section{Internal Charge Accumulation in Glass Materials}

Especially, we focused on an accident of spacecraft caused by internal charging in a glass material that was used as the cover plate of solar array. Therefore we tried to measure the charge accumulation in glass materials under electron beam irradiation by using a PEA method [12]. It was obtained two unexpected results that no charge accumulation in quartz glass (pure $\mathrm{SiO}_{2}$ ) was observed under the electron beam irradiation and the positive charge accumulation was observed in glass samples containing metal-oxide compound [13].

Table 1 shows the chemical composition of glass materials used in this experiment. The basic component of these glass samples is $\mathrm{SiO}_{2}$. Alumina $\left(\mathrm{Al}_{2} \mathrm{O}_{3}\right)$ is added to improve their electric property, while metal-oxide compounds such as oxide calcium ( $\mathrm{CaO})$ and oxide sodium $\left(\mathrm{Na}_{2} \mathrm{O}\right)$ are added to decrease their melting points. The samples thickness are about $1.0 \mathrm{~mm}$. Samples have evaporated aluminum electrodes on both surfaces.

\section{Negative and positive charge accumulation}

Figure 12 (a) shows the negative charge distributions accumulated after the e-beam irradiation in T-glass. Since the both electrodes on surfaces of the sample are grounded during the measurements, the interfacial charges are induced at electrodes with opposite polarity to the accumulated charge in bulk of sample. Figure 12 (b) shows the space charge distribution in E-glass. Even though E-glass was irradiated by negative electron beams, positive charge was observed to accumulate. Corresponding to these positive charges, negative charges were induced at the grounding electrode surface on the irradiation side. Up till now, negative charges were always observed to accumulate in polymer insulating materials such as PMMA under electron beam irradiation. It was the first time to observe the accumulation of positive charges as that in E-glass.

Now, it is speculated that the alumina $\left(\mathrm{Al}_{2} \mathrm{O}_{3}\right)$ in T-glass plays the role of trap center for capturing electrons and contributes to the negative charge accumulation, while the metal-oxide compound of alkali $(\mathrm{CaO}, \mathrm{Na} 2 \mathrm{O}$ ) in $\mathrm{E}$-glass plays the role of releasing electrons and contributes to the positive charge accumulation.

\section{Charge accumulation in quarte glass and PMA}

When the glass materials including some additives were irradiated by negative electron beam, some showed the positive charge accumulation and others showed the negative in the bulk. Since it was thought that the difference in the polarity of accumulated charges depended on the additives (metal-oxide compounds) in the glass materials, the charge distributions in pure quartz glass $\left(\mathrm{SiO}_{2}\right)$ and polymer PMMA without any metal-oxide compounds were measured 
Table 1. Chemical composition of glass samples
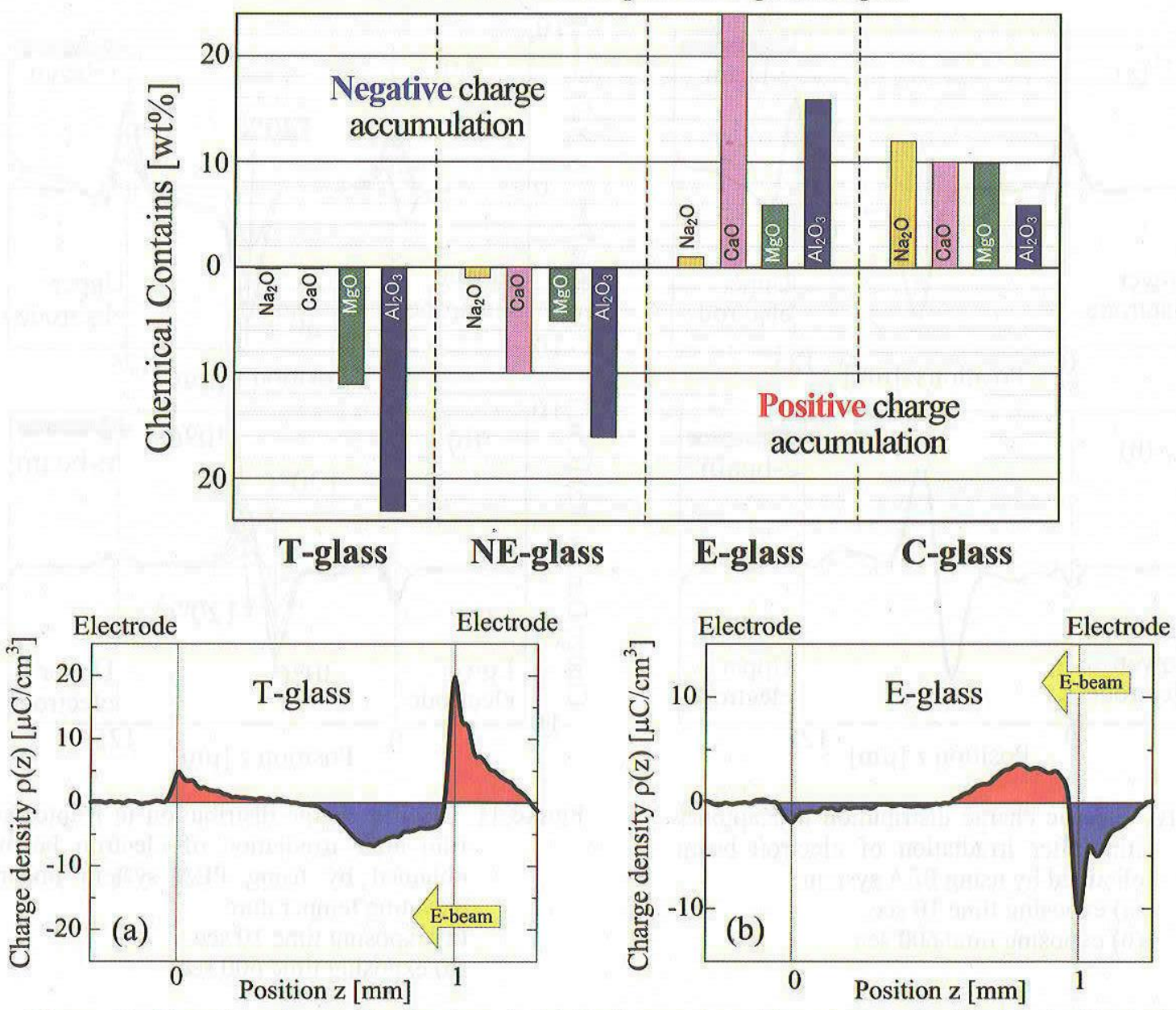

Figure 12. Negative charge distributions in (a) T-glass and positive charge distribution in (b) E-glass after the e-beam irradiation
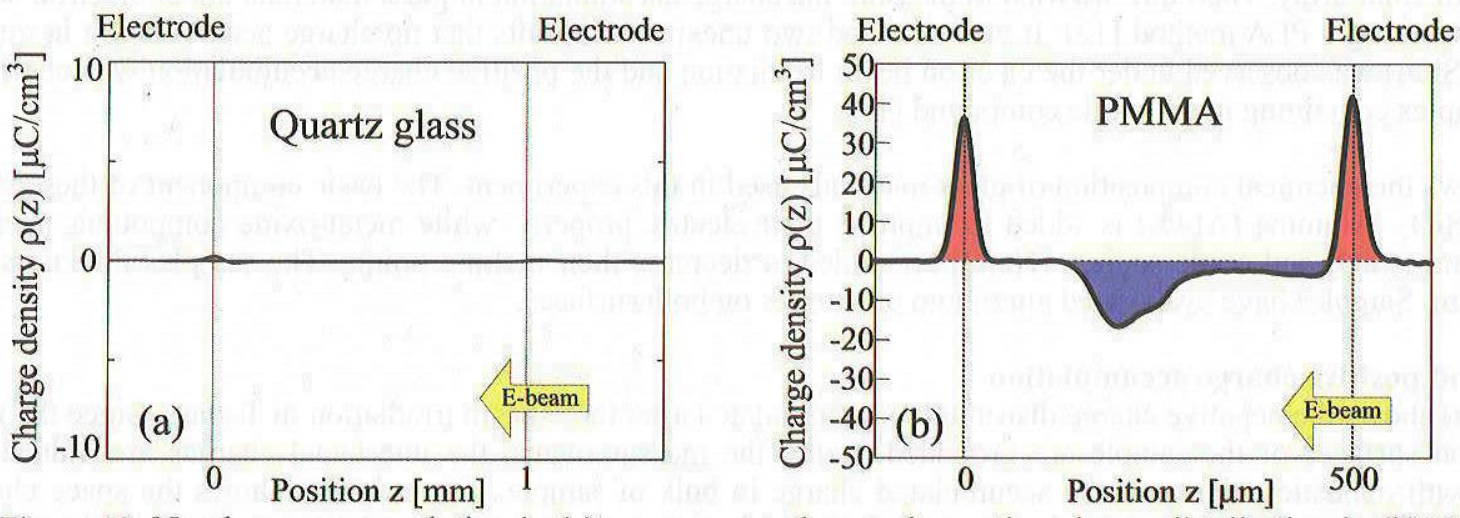

Figure 13. No charge accumulation in (a) pure quartz glass and negative charge distribution in (b) PMMA during electron beam irradiation.

during and after e-beam irradiation.

The results of the charge distribution measurements in both samples are shown in Figure 13(a) and (b), respectively. As shown in Figure13 (a), no remarkable charge is observed in the pure quartz glass neither during nor after the electron beam irradiation. On the other hand, as shown in Figure 13 (b), a large amount of negative charge is observed in PMMA, which is not containing any metal-oxide compounds. The amount and polarity of accumulated charges remained in glass materials by e-beam irradiation are considered to depend on the composition of metal-oxide compounds included in the glass materials.

As shown in above results, since the charge accumulation in glass materials strongly depends on the chemical compounds, we have to study more about the relationship between the charge accumulation and the chemical compounds. 


\section{Internal Charge Accumulation by Gamma Ray Irradiation}

As you know the gamma ray and $\mathrm{X}$ ray is one of electro-magnetic wave and they are neutral electrically. However, an ionization ability of gamma ray is very high. When the dielectric material is irradiated by gamma ray, a lot of pair of positive parent ion and negative electrons are expected to be generated and to be distributed uniformly in the material. Therefore we attempted to observe the charge accumulation in dielectrics after gamma ray irradiation under dc stress.

\section{Results and Discussion}

Figure 14 shows the charge accumulation process in LDPE (Low-density polyethylene) after gamma ray irradiated under dc voltage stress. The charge distributions were measured using PEA method. The thickness of LDPE is about $630 \mu \mathrm{m}$ and the applied voltage is $15 \mathrm{kV}$. The sample was irradiated by gamma ray from $60 \mathrm{Co}$ source with dose rate of $12 \mathrm{~Gy} / \mathrm{h}$ and total dose of $2.4 \mathrm{kGy}$ in air atmosphere. Before voltage application, no charge was observed in the bulk even LDPE sample was irradiated by gamma ray. After voltage application, the positive charge appeared near cathode developed towards the anode, and then the charge distribution becomes stable, finally, as shown in Figure 14 (a). Figure 14 (b) shows a time dependent charge distribution under short circuit condition after voltage application. After the voltage on, the positive charge near cathode decreased gradually, and another positive charge appeared near anode.

Analysis model of the charge accumulation and decay process mentioned above is considered in following. Consider electrons and parent positive ions with density $\rho$, which is escaped from recombination, remained and uniformly distributed in the in gamma ray irradiated LDPE sample. Here, we assume if dc voltage is applied to this LDPE sample, only the electrons are mobile, and there is no injecting charge from electrode. When some of the electrons are drifted and swept out into an anode by the applied dc voltage, some of positive ions become observable. Details of analysis model are described in elsewhere [13].

\section{Internal Charge Accumulation by Proton Irradiation}

Since the high energy proton is one of radioactive rays in space environment, the positive charge accumulation in dielectric materials irradiated by proton beam irradiation should be investigated. We have carried out the observation of charge distribution in insulating material irradiated by proton beam. The energy values of $1.0,3.0$ and $6.0 \mathrm{MeV}$ were
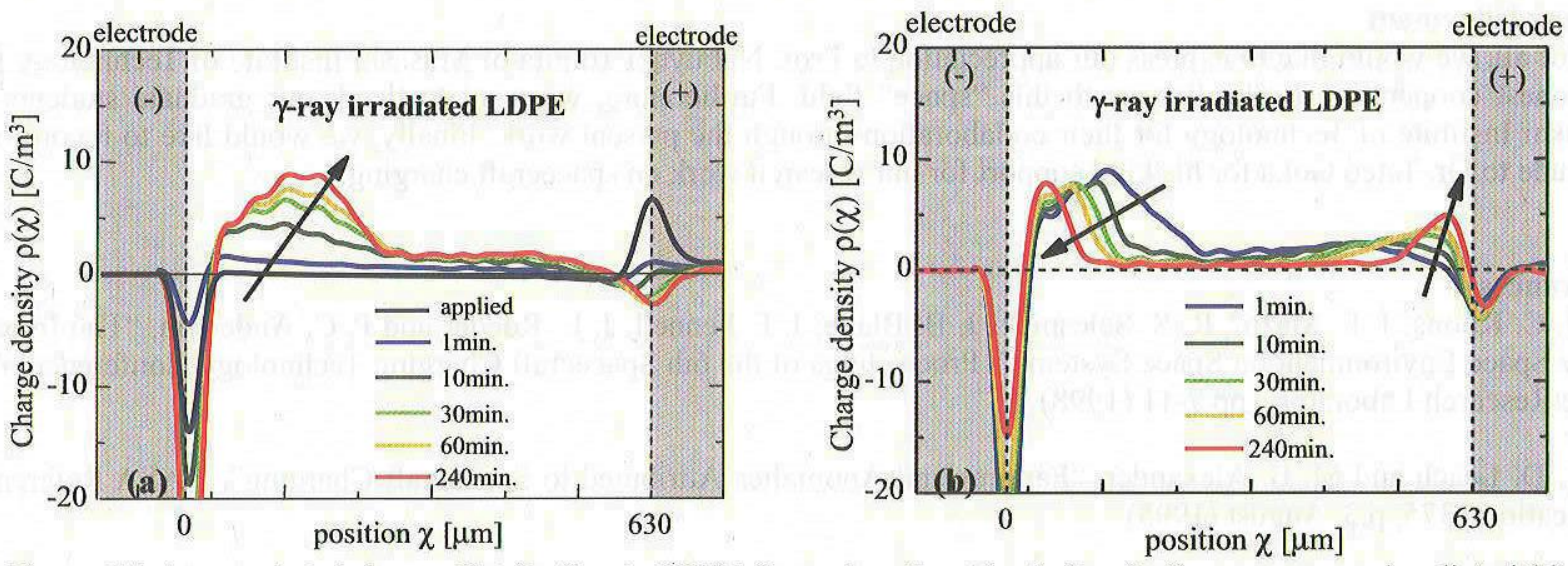

Figure 14. Accumulated charge distribution in LDPE (Low-density polyethylene) after gamma ray irradiated (a) under dc voltage stress and (b) under short circuit condition after voltage application

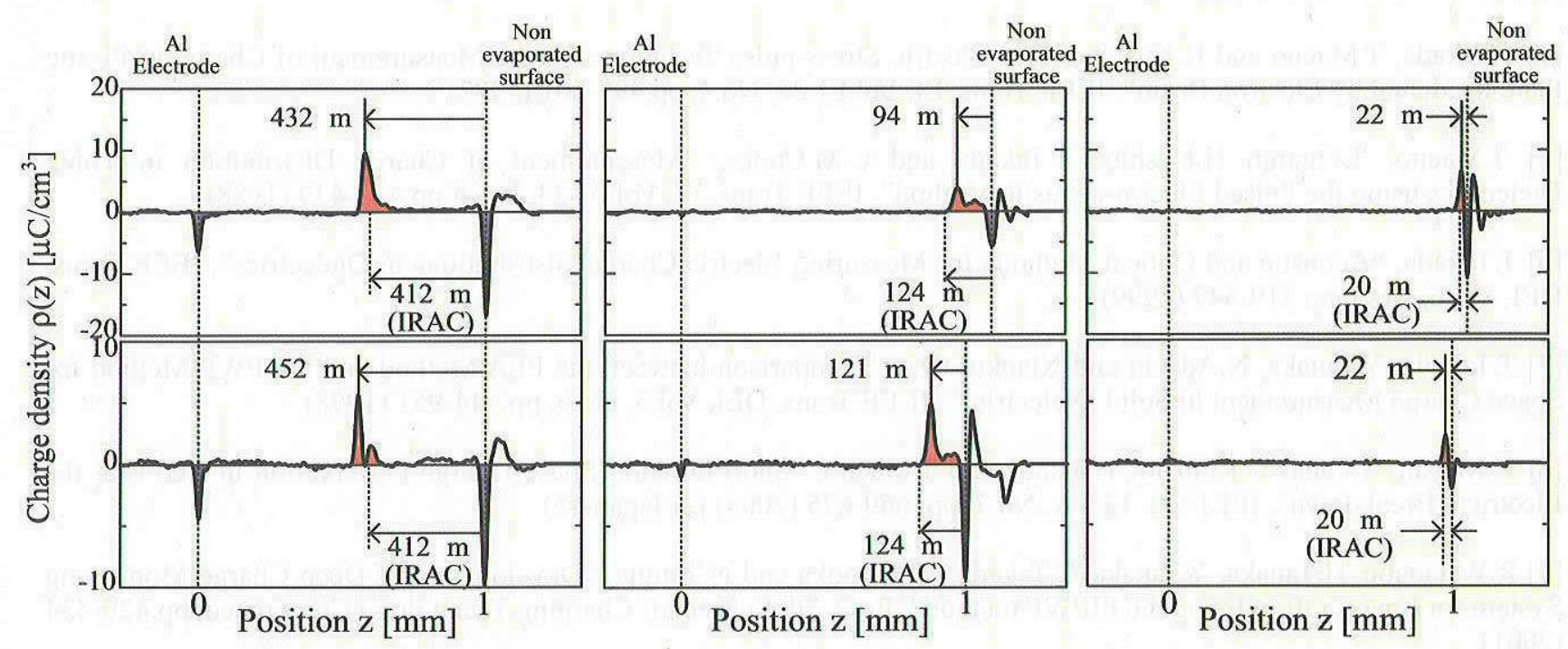

Figure 15. Positive charge distribution irradiated by proton beam in PMMA sample 
adopted because the energy of proton in space environment is said from 1.0 to $6.0 \mathrm{MeV}$. The irradiation current density of proton beam was $30 \mathrm{nA} / \mathrm{cm}^{2}$ and the exposing time was $180 \mathrm{sec}$. The test sample was PMMA with thickness of 1.0 mm. The charge distribution in proton beam irradiated PMMA was measured using PEA method after irradiation.

\section{Resulles and Discenssion}

Figure 15 shows the charge distribution in proton irradiated PMMA. The signal of positive charge accumulation in the sample was observed at the middle of sample. The negative charges show the induced charge at both ground electrodes. They are induced by accumulated positive charge in the bulk. The peak positions of positive charges are 432,94 and 22 $\mu \mathrm{m}$ from irradiation surface with irradiation energy of $6.0,3.0$ and $1.0 \mathrm{MeV}$, respectively. The penetration depths of proton in PMMA were calculated using a simulation model [14]. The values of them are 412, 124, 20um for 6.0, 3.0 and 1.0 MeV, respectively. The calculated values are very close to the peak position of positive charge shown in Figure 15.

\section{Concinsion}

In this century, the size of spacecraft must be larger and larger, because the spacecraft are demanded for the multi functions of broadcasting, communication, whether observation, and so on. It seems that the probability of accident in spacecraft caused by high energy radioactive rays becomes higher. With increase of the size of spacecraft, the sizes of dielectric materials on spacecraft such as the cover of solar array panel, the insulator for wire harness and/or thermal blanket becomes large. In other words, the area where is exposed by high energy radioactive rays becomes large with increase of the size of spacecraft. Therefore, it seems to be more and more important to investigate the internal and surface charging properties of dielectric materials for spacecraft.

As you know, all administration, NASA, JAXA, ESA and CAST, have accumulated a lot of experience for spacecraft charging technology. On the other hand, our group, Musashi Institute of Technology, Tokyo, has an experience to develop both acoustic and optic measurement techniques to observe the internal and the surface charge accumulation. Now we have an excellent tool of PEA and PWP methods for evaluating electric charge accumulation properties. We would like to propose the collaboration program of "Solution for Spacecraft Charging Issues".

\section{Acknowledgement}

First of all, we would like to express our appreciation to Prof. Nobuyuki Tomita of Musashi Institute of Technology for his honest cooperation by leading us to this "space" field. Furthermore, we want to thank our graduate students at Musashi Institute of Technology for their collaboration through the present work. Finally, we would like to record our gratitude to Dr. Tateo Goka for his kind support for our research work on spacecraft charging.

\section{Refererences}

[1] H. C. Koons, J. E. Mazur, R. S. Selesnick, J. B. Blake, J. F. Fennell, J. L. Roeder and P. C. Anderson, "The Impact of the Space Enviromment on Space Systems", Proceedings of the 6th Spacecraft Charging Technology Conference, Air Force Research Laboratory, pp.7-11 (1998)

[2] R. D. Leach and M. B. Alexander, "Failures and Anomalies Attributed to Spacecraft Charging", NASA Reference Publication 1375, p.3, August (1995)

[3] S. Hiro and H. Fujii, "Electron-Beam-Induced Charging of Polymer Films for Space Use", IEEJ Vol. 117-A, No.8, pp. 805-812 (1997) (in Japanese)

[4] T.Takada, T.Maeno and H.Kushibe, "An Electric Stress-pulse Technique for the Measurement of Charge in Plastic Plate Irradiated by Electron Beam", IEEE Trans. EI, Vol.EI-22, No.4, pp.497-501 (1987)

[5] T.Maeno, T.Futami, H.Kushibe T.Takada and C.M.Cooke, "Measurement of Charge Distribution in Thick Dielectrics using the Pulsed Electro-acoustic Method", IEEE Trans. EI, Vol.EI-23, No.4, pp.433-439 (1988)

[6] T.Takada, "Acoustic and Optical Methods for Measuring Electric Charge Distributions in Dielectrics", IEEE Trans. DEI, Vol.6, No.5, pp.519-547 (1999)

[7] T.Takada, Y.Tanaka, N.Adachi and Xiaokui Qin, "Comparison between the PEA Method and the PWP Method for Space Charge Measurement in Solid Dielectrics", IEEE Trans. DEI, Vol.5, No.6, pp.944-951 (1998)

[8] K.Matsui, Y.Tanaka, T.Fukao, T.Takada and T.Maeno, "Short-duration Space Charge Observation in LDPE at the Electrical Breakdown", IEEJ Vol. 123-A, No.7, pp. 669-675 (2003) (in Japanese)

[9] R.Watanabe, H.Tanaka, Y.Tanaka, T.Takada, Y.Murooka and N.Tomita, "Development of Deep Charge Monitoring System for Spacecraft utilizing the PIPWP method", Proc. 7th Spacecraft Charging Technology Conference, pp. $429-434$ (2001) 
[10] Y. Ohkami, N. Tomita, S. Nakasuka and S. Matsunaga, "Introduction to the Space Station", University of Tokyo Press, Section 11, p. 251 (2002) (in Japanese)

[11] Y.Tanaka, H.Kitajima, M.Kodaka and T.Takada, "Analysis and Discussion on Conduction Current Based on Simultaneous Measurement of TSC and Space Charge Distribution", IEEE Trans. DEI, Vol.5, No.6, pp.952-956 (1998)

[12] S. Le Roy, H. Miyake, Y Tanaka, T Takada, G Teyssedre and C Laurent, "Simultaneous Measurement of Electroluminescence and Space Charge Distribution in Low Density Polyethylene under Uniform DC Field", J. Phys. D: Appl. Phys. 38, Issue 1, pp.89-94 (2005)

[13] H. Miyake, Y. Tanaka and T. Takada: "Characteristic of Charge Accumulation in Glass Materials under Electron Beam Irradiation", Proceedings of 8th Spacecraft Charging Technology conference, NASA/CP-2004-213091, Ground Testing Techniques Section, No.8 (2003)

[14] M.Kojima, Y.Tanaka, T.Takada and Y.Ohki, "Measurement of Space Charge Accumulation in Gamma-irradiated Polyethylene with DC Voltage" Trans. IEE of J, Vol.115-A, No.2, pp.93-98 (1995)

[15] S. Tanaka, M. Fukada, K. Nishimura, W. Yokota, T. Kamiya and H. Watanabe, "Development of IRAC code system to calculate induced radioactivity produced by ions and neutrons", Proceedings 8 th International Conference on Radiation Shielding, Vol. 2, pp. 965-971 (1994) 